\title{
Google Street View como herramienta docente: Una actividad formativa desde el Research Based Learning
}

\author{
Miriam Marcoa, Viviana Vargas ${ }^{b}$, Manuel Martín-Fernández ${ }^{c}$, Faraj A. Santirsod, \\ Enrique Gracia ${ }^{\mathrm{e}}$ y Marisol Lila \\ aDepartamento de Psicología Social. Universitat de València. E-mail: Miriam.Marco- \\ Francisco@uv.es, ${ }^{b}$ Departamento de Psicología Social. Universitat de València. E-mail: \\ vivianav@gmail.com, ${ }^{c}$ Departamento de Psicología Social. Universitat de València. E-mail: \\ Manuel.Martin@uv.es, ${ }^{\mathrm{d}}$ Departamento de Psicología Social. Universitat de València. E-mail: \\ Faraj.Santirso@uv.es, e Departamento de Psicología Social. Universitat de València. E-mail:

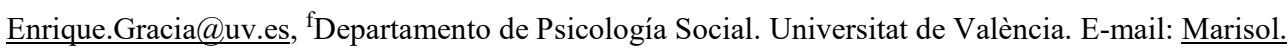 \\ $\underline{\text { Lila@,uv.es }}$
}

\begin{abstract}
From the Research Based Learning approach, it has been highlighted the importance of introducing new teaching tools in order to let students to acquire practical knowledge about a subject from a scientific perspective. This paper presents a structure practice developed and applied in the subject of Psychology of Social and Community Intervention of the Degree of Psychology (University of Valencia). "Learning by doing. Using Google Street View as a research tool" is a practice where students use the tool Google Street View to assess the neighborhood disorder after which they get feedback of the result of their work. This activity promotes the debate, and then a work by projects is presented, where students have to design a community intervention based on the concepts learned in the practice. The proposed activity had a very positive response from the students due to the novelty of the concept, the introduction of new technological tools such as Google Street View, and the autonomous work by students. Future research would benefit from assessing the effectiveness of this practice.
\end{abstract}

Keywords: Research Based Learning, Learning by doing, Google Street View, new teaching tools

\section{Resumen}

Desde la perspectiva del Research Based Learning se ha destacado la importancia de introducir nuevas herramientas docentes que permitan a los estudiantes adquirir conocimientos prácticos sobre una materia desde un punto de vista científico, conectando con la investigación. En este trabajo se presenta una práctica estructurada desarrollada y aplicada en la asignatura de Psicología de la Intervención Social y Comunitaria del Grado de Psicología de la Universidad de Valencia. "Aprender haciendo. La utilización de Google Street View como herramienta de investigación" es una práctica que consiste en que los alumnos utilicen la herramienta Google Street View para evaluar el desorden del vecindario y posteriormente obtengan retroalimentación del resultado de su trabajo. Con esta actividad 
se fomenta el debate, y posteriormente se presenta un trabajo por proyectos donde los estudiantes deben diseñar una intervención comunitaria basada en los conceptos vistos en la práctica. La actividad propuesta tuvo una respuesta muy positiva por parte de los alumnos por lo novedoso del concepto, la introducción de nuevas herramientas tecnológicas como Google Street View, y la capacidad de trabajo autónomo por parte de los alumnos. Futuros estudios son necesarios para evaluar la efectividad de dicha práctica.

Palabras clave: Research Based Learning, Aprender Haciendo, Google Street View, nuevas herramientas docentes 


\section{Introducción}

Tradicionalmente la investigación y la docencia se han tratado como dos funciones independientes y diferenciadas en el ámbito universitario. Sin embargo, tanto para docentes como para los y las estudiantes, es importante integrar estos dos aspectos y considerarlos como parte de un todo: la teoría no avanza ni evoluciona si no se basa en la investigación científica, mientras que la investigación no es válida si no se basa en el conocimiento teórico. Es por ello por lo que en las últimas décadas el trabajo académico universitario consiste en combinar ambos perfiles, dando lugar a una práctica integradora que se traduce en el papel del docente-investigador.

Desde este punto de vista, los alumnos, que están inmersos en el sistema universitario, deben ser capaces de comprender la importancia que la investigación tiene para el avance del conocimiento científico. Así como también, deben conocer las oportunidades que tienen de ser parte de este avance a través de la generación de su propio conocimiento científico (Lee, 2004). Por ello, es necesario que desde la docencia universitaria se fomente el interés de los alumnos en la investigación y en todo lo que ésta supone (Badley, 2002; Elton, 2001).

Desde la perspectiva del Research Based Learning, se propone la importancia de introducir nuevas herramientas docentes que permitan a los estudiantes adquirir conocimientos prácticos sobre una materia concreta desde un punto de vista científico, vinculándolo con la investigación y el avance del conocimiento (Griffits, 2004; Hutchings, 2007; Jenkins, 2004). Técnicas como los estudios de caso, el trabajo de campo, o los trabajos por proyectos formarían parte de esta nueva perspectiva (Healey y Jenkins, 2009).

\begin{tabular}{|c|c|c|c|}
\hline \multirow{6}{*}{$\begin{array}{l}\text { ÉNFASIS EN EL } \\
\text { CONTENIDO DE } \\
\text { INVESTIGACIÓN }\end{array}$} & \multicolumn{2}{|c|}{ LOS ESTUDIANTES PARTICIPAN } & \multirow{5}{*}{$\begin{array}{l}\text { ÉNFASIS EN LOS } \\
\text { PROCESOS Y Y } \\
\text { PROBLEMAS DE } \\
\text { INVESTIGACIÓN }\end{array}$} \\
\hline & Research-tutored & Research-based & \\
\hline & $\begin{array}{c}\text { Participar en } \\
\text { discusiones sobre } \\
\text { investigación }\end{array}$ & $\begin{array}{c}\text { Llevar a cabo } \\
\text { investigaciones }\end{array}$ & \\
\hline & Research-led & Research-oriented & \\
\hline & $\begin{array}{l}\text { Aprender sobre la } \\
\text { investigación } \\
\text { actual en la } \\
\text { materia }\end{array}$ & $\begin{array}{l}\text { Desarrollar } \\
\text { habilidades y } \\
\text { técnicas de } \\
\text { investigación }\end{array}$ & \\
\hline & LOS ESTUDIAN & SON OYENTES & \\
\hline
\end{tabular}

Fig. 1 La naturaleza del aprendizaje basado en la investigación (fuente: Healey y Jenkins, 2009) 
Partiendo de esta base, se ha diseñado un proyecto docente con el objetivo de acercar la investigación a las aulas universitarias. Para ello, se ha establecido un plan formativo estructurado en el que se han desarrollado diferentes actividades o prácticas donde se introduce la investigación en este contexto. En este trabajo nos vamos a centrar en una de esas actividades en concreto, exponiendo las bases de esta práctica, y la forma en que se ha preparado e impartido.

\section{Objetivos}

\subsection{Objetivo general}

En este trabajo se presenta una actividad docente que se ha desarrollado y se ha aplicado en la asignatura de Psicología de la Intervención Social y Comunitaria del Grado de Psicología de la Universidad de Valencia. El objetivo general de la actividad es fomentar la curiosidad científica de los alumnos, desde una perspectiva basada en el Research Based Learning.

\subsection{Objetivos específicos}

- Introducir herramientas de Aprender Haciendo como alternativa a la enseñanza tradicional.

- Presentar a las y los alumnos la importancia del contexto social y estructural del vecindario a la hora de estudiar distintos problemas sociales.

- Presentar a las y los alumnos el concepto de desorden del vecindario y enseñar un instrumento de observación válido y preciso del mismo.

- Fomentar el aprendizaje acerca de los procesos sociales y la intervención en la comunidad mediante trabajo de campo.

- Favorecer el trabajo en equipo y la discusión sobre los resultados científicos.

\section{Desarrollo de la innovación}

La práctica que se expone en este trabajo tiene por nombre "Aprender haciendo. La utilización de Google Street View como herramienta de investigación”. Como se ha comentado anteriormente, esta actividad docente se diseñó para llevarse a cabo dentro de la asignatura de Psicología de la Intervención Social y Comunitaria, dentro del Grado de Psicología. Esta actividad docente de innovación educativa consiste en presentar a los alumnos un instrumento para medir el desorden del vecindario (una variable especialmene relevante en el estudio de diferentes problemas sociales) mediante la aplicación tecnológica Google Street View. En la actualidad, esta herramienta es cada vez más utilizada para evaluar características del vecindario por las grandes ventajas que presenta (Odgers, Caspi, Bates, Sampson, y Moffit, 2012). A continuación, se describe la actividad principal de la práctica propuesta, consistente en que las y los alumnos utilicen dicha herramienta para la

(cc) EY-NC-ND 2017, Universitat Politècnica de València 
evaluación del desorden social en los vecindarios. Posteriormente, son presentados en conjunto los resultados obtenidos por los/as alumnos/as, y se muestran mapas de desorden del vecindario obtenidos a partir del trabajo de cada uno de ellos/as.

\subsection{Prueba piloto}

Se llevó a cabo un estudio piloto en el que se realizó esta práctica en el curso académico 2015-2016 en varios grupos de la asignatura de Psicología de la Intervención Social y Comunitaria como una actividad incluida en el plan docente de la asignatura. El estudio piloto fue desarrollado en dos sesiones presenciales realizadas en el aula y una sesión no presencial en la que los alumnos debían realizar una tarea en casa. En la primera sesión presencial se les explicó a los/as alumnos/as el contenido de la práctica, así como la importancia del contexto y de las variables contextuales en el estudio de diferentes problemas sociales. Además se presentó el concepto teórico de desorden del vecindario. A continuación, se presentaron algunos instrumentos de observación realizados anteriormente por investigadores para la valoración del desorden social (Marco, Gracia, Tomás y LópezQuílez, 2015). Sin embargo, debido a los inconvenientes de estos instrumentos observacionales, los investigadores han comenzado a utilizar en mayor medida herramientas tecnológicas, entre estas la aplicación Google Street View. Asimismo, se les explicó en qué consistía esta herramienta, y se realizó una práctica en la que los/as alumnos/as debían evaluar entre todos los diferentes ítems de desorden del vecindario contenidos en una escala de observación. Los/as alumnos/as ante cada ítem debían llegar a un acuerdo con respecto a la puntuación. Una vez alcanzado un alto grado de consenso, se repartieron entre los/as alumnos/as diferentes áreas de Valencia, las cuales debían evaluar en parejas utilizando el Google Street View.

Una vez concluída la sesión presencial, los y las estudiantes debían realizar por parejas la evaluación del área asignada durante una sesión no presencial. Para ello se les pidió que seleccionaran aquellas fotos virtuales que creyeran más representativas del desorden del vecindario de las áreas que tenían asignadas. Asimismo, se les pidió que llegasen a un acuerdo y evaluaran el desorden general del vecindario sobre la base de sus observaciones. Una vez hubieran terminado con la evaluación, debían enviar el resultado a los docentes a través del aula virtual de la asignatura.

Por último, en la segunda sesión presencial la docente mostró a toda la clase los resultados de los trabajos realizados por los/as alumnos/as. Entre los resultados se presentaron algunas características psicométricas de la escala, así como mapas de desorden del vecindario según la valoración de los propios alumnos/as. Estos resultados fueron comparados con los obtenidos anteriormente mediante una escala observacional física donde se visitó de forma directa las áreas y se evaluaron mediante observaciones in situ (Marco et al., 2015). 


\subsection{Desarrollo de la práctica}

Una vez realizado el estudio piloto, se procedió a diseñar la práctica de forma sistematizada y crear fichas de contenido estructuradas para cada una de las sesiones de la práctica. Se planificó 3 sesiones para abarcar todo el contenido necesario. A continuación, se describe el desarrollo de la práctica en las 3 sesiones tal y como se muestra en las fichas de contenido.

\subsubsection{Sesión 1}

Objetivos:

- Presentar a los alumnos la importancia del contexto y el vecindario a la hora de estudiar distintos problemas sociales

- Presentar a los alumnos el concepto de desorden del vecindario

- Enseñar un instrumento de observación de desorden del vecindario

- Realizar una práctica guiada sobre cómo evaluar el desorden del vecindario mediante Google Street View utilizando la escala observacional presentada

- Llegar a un alto nivel de acuerdo entre los alumnos en cuanto a la evaluación de desorden del vecindario

Duración: 120 minutos

Material del docente: Diapositivas power point, instrumento de desorden del vecindario.

Dinámica de trabajo: Exposición teórica y trabajo en gran grupo

Procedimiento:

- El docente explica mediante power point la importancia del contexto y el vecindario a la hora de estudiar distintos problemas sociales poniendo ejemplos específicos: delitos, violencia de género, maltrato infantil, etc. (15 min.).

- Seguidamente, el docente explica las diferentes características contextuales que se han estudiado, y presenta el concepto de desorden del vecindario y las teorías que lo explican (15 min).

- El docente explica las diferentes formas que se han utilizado para evaluar el desorden del vecindario, y se explica qué son los instrumentos observacionales y su utilidad (15 min).

- El docente presenta un instrumento de observación validado realizado por el grupo docente que evalúa desorden del vecindario (Marco et al., 2015). El instrumento contiene 6 ítems de desorden físico (colillas y basura en la calle, botellas o latas vacías, graffitis, coches abandonados, y pintadas de carácter político o reivindicativo) y 5 ítems de deterioro físico (casas vacías, comercios o locales abandonados, viviendas abandonadas, zonas residenciales muy deterioradas, y zonas recreativas muy deterioradas). (15 min.).

- A continuación, se les explica a los alumnos la herramienta de Google Street View, su modo de funcionamiento, sus ventajas respecto a las observaciones directas y se presentan algunos estudios que lo han utilizado (15 min.).

(cc) EY-NC-ND 2017, Universitat Politècnica de València 
- Se realiza una práctica guiada sobre cómo evaluar el desorden del vecindario mediante Google Street View utilizando la escala observacional presentada anteriormente Cada uno de los alumnos sale al ordenador de clase a realizar la práctica, el docente le pide que recorra un área concreta y que comente a los compañeros lo que va encontrando, y finalmente proponga una puntuación a los diferentes ítems de la escala. Entre todos los alumnos han de llegar a un acuerdo sobre la puntuación para cada ítem. Si existen discrepancias entre los alumnos, llegar a un punto en común y asumir normas que se van a seguir siempre que se dé la misma situación.El docente anticipa los posibles problemas que puedan surgir en la visualización, y cómo solventarlos. Se practica en conjunto hasta que todos los ítems han quedado claros y los alumnos llegan a un alto grado de acuerdo (45 min.).

- Antes de la finalización de la sesión, se recuerda a los alumnos que la semana siguiente deben volver a traer el ordenador.

\subsubsection{Sesión 2}

Objetivos:

- Que los alumnos, en parejas, realicen la evaluación de una zona concreta (diferentes sectores censales de la ciudad) y rellenan los diferentes ítems del cuestionario de evaluación de desorden

- Que los alumnos lleguen a un alto nivel de acuerdo sobre las puntuaciones. Además, deben hacer capturas de pantalla sobre los elementos más relevantes del área correspondiente, y escribir las observaciones que crean oportunas

Duración: 120 horas

Material de los alumnos: Web de Google, instrumento de desorden del vecindario y mapa de la ciudad de Valencia.

Materiales del docente: Web de Google.

Dinámica de trabajo: Trabajo en parejas.

Procedimiento:

- El docente explica que hoy se va a realizar la práctica de Google Street View. Los alumnos se ponen por parejas, y el docente les reparte un mapa de la ciudad de Valencia (ver Figura 1). Cada pareja de la clase tendrá un mapa diferente, dividido en sectores censales. Se explica que deben completar el instrumento de desorden del vecindario para cada uno de los sectores censales que contenga su mapa, y deben realizar una pequeña ficha sobre cada uno de ellos, las observaciones que quieran poner, y fotos virtuales sobre las zonas más características del sector censal en concreto (15 min.).

- Los alumnos trabajan en parejas durante la clase. Si tienen dudas, el docente les ayuda a la consecución del trabajo de manera correcta (1 h. y 45 min.). 
- En caso de que no les dé tiempo a acabar, podrán continuar el trabajo fuera del aula durante la semana, y deberán entregar la actividad mediante el aula virtual de la asignatura en los dos días siguientes.

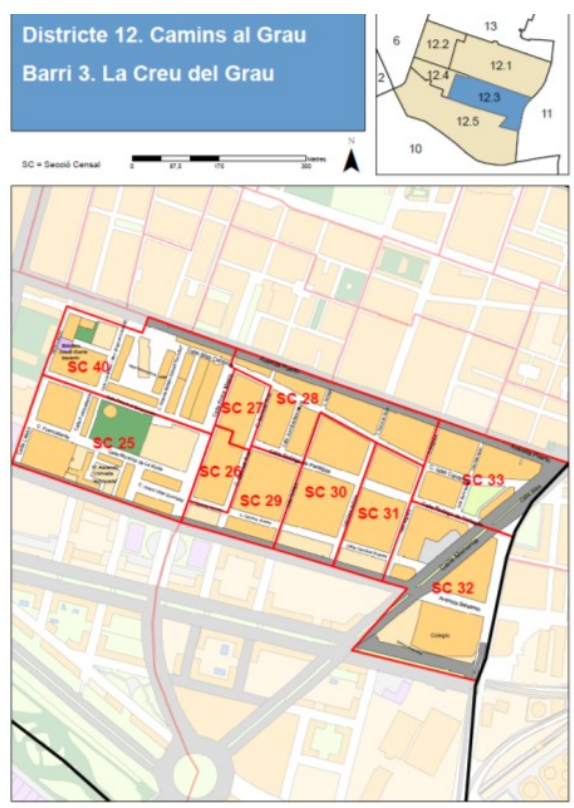

Fig. 2 Ejemplo de mapa de trabajo

\subsubsection{Sesión 3}

Objetivos:

- Presentar a los alumnos los resultados de sus análisis para que vean el producto de su trabajo

- Analizar con ellos las características psicométricas del instrumento de desorden del vecindario mediante el uso de Google Street View

Duración: 120 horas

Materiales del docente: Diapositivas power point.

Dinámica de trabajo: Exposición del docente.

Procedimiento:

- El docente comienza la clase recapitulando lo que se ha hecho hasta ahora y agradeciendo a los alumnos por su trabajo en las sesiones anteriores (10 min.).

- A continuación, el docente muestra a los alumnos los resultados de sus análisis: enseñará la fiabilidad del instrumento (si se han obtenido resultados similares a los obtenidos con observaciones in situ), el grado de acuerdo entre los alumnos, y mostrará los mapas de desorden que se han podido crear gracias al trabajo de los alumnos (30 $\mathrm{min}$.).

(c)) EY-NC-ND 2017, Universitat Politècnica de València 
- El docente presenta algunas de las fotos virtuales realizadas por los alumnos más significativas, y las compara con fotografias hechas in situ para mostrar la similitud entre ambas (15 min.)

- Por último, el docente abre un debate entre los alumnos donde se comente por qué el desorden del vecindario puede ser tan importante para este tipo de problemas sociales, y planteen de qué forma podrían intervenir para disminuir el desorden del vecindario en las áreas donde más desorden se ha encontrado. Para ello, se realiza una actividad grupal donde los alumnos ideen una intervención comunitaria en un barrio con altos niveles de desorden físico y otras características negativas del vecindario, y presenten su proyecto de forma oral llevando a cabo estrategias de márketing social (50 min.).

\section{Resultados}

La primera puesta en práctica sistematizada de la práctica "Aprender haciendo. La utilización de Google Street View como herramienta de investigación" se llevó a cabo durante el primer cuatrimestre del curso 2016-2017 en un total de 3 grupos de la asignatura de Psicología de la Intervención Social y Comunitaria. En este apartado se muestran algunos de los resultados obtenidos por los alumnos, que fueron compartidos y discutidos en la última sesión.

En la Figura 2, se muestran ejemplos de las fotografías realizadas por los alumnos que fueron presentadas en la sesión.
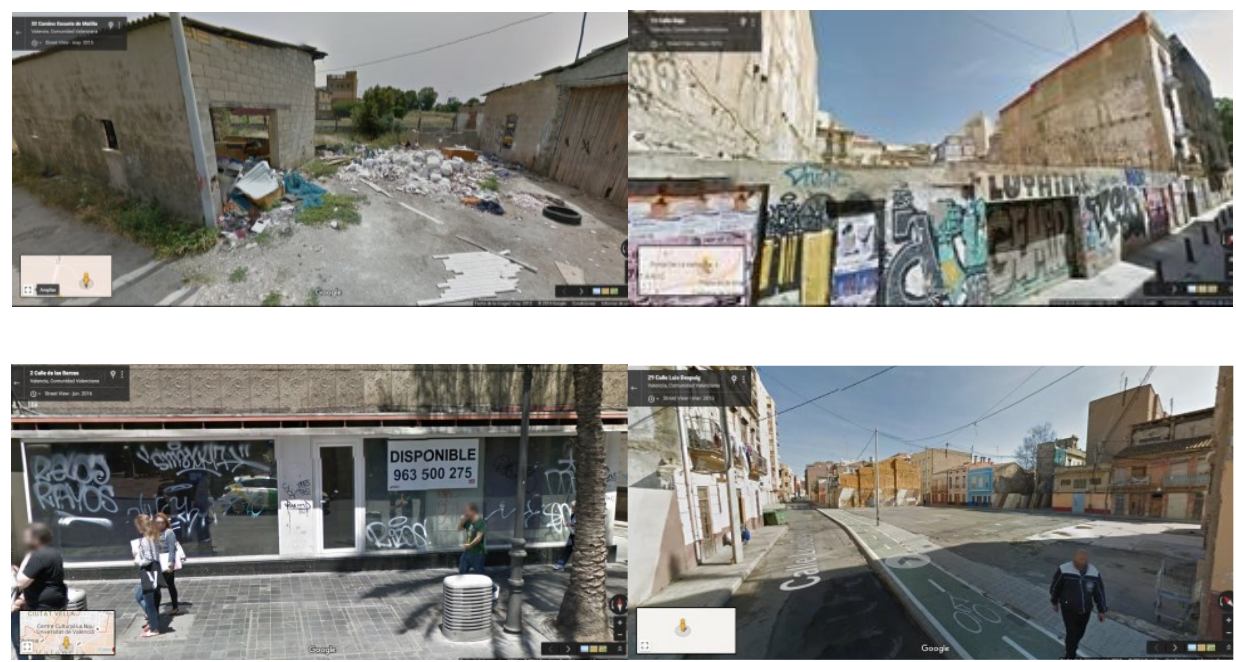

Fig. 3 Ejemplos de los alumnos sobre los items Basura en la calle, Grafitis, Locales Abandonados y Zonas residenciales muy deterioradas respectivamente, recogido con Google Street View 
Además, se les presentó una comparativa entre las fotografías tomadas por ellos mediante Google Street View, y fotografías in situ de las mismas áreas (Figura 3).

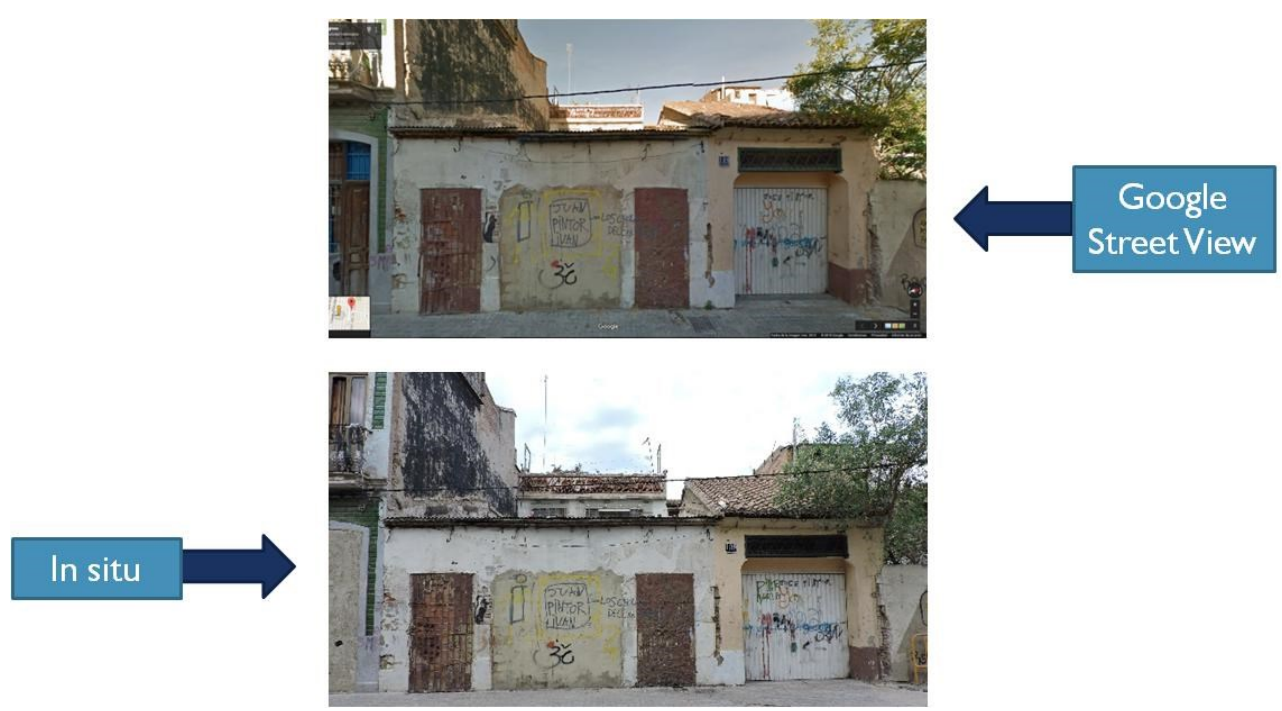

Fig. 4 Comparativa entre una fotografia tomada mediante Google Street View por los alumnos y una fotografia in situ

A continuación, se presentaron los diferentes análisis y mapas que mostraban visualmente la similitud entre las puntuaciones totales de deterioro físico y desorden físico obtenidas por los alumnos y alumnas, y las puntuaciones obtenidas en una evaluación in situ en la misma área (Marco et al., 2015) (Figura 4).
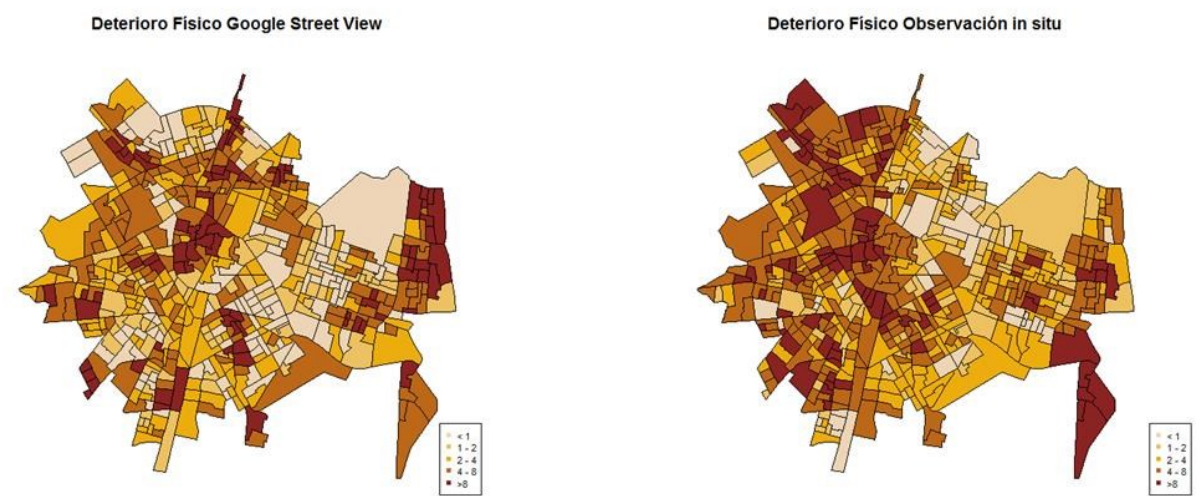

Fig. 5 Ejemplo de mapa presentado a los alumnos comparando las puntuaciones de desorden obtenidas mediante Google Street View y las obtenidas en observación in situ. 
Después, se discutió en grupos acerca de los resultados obtenidos, analizando las áreas que, según sus observaciones, presentaban mayores niveles de desorden, y las dificultades que habían tenido a la hora de realizar la observación virtual.

A continuación, se generó un debate entre los alumnos sobre la importancia de este tipo de variables para los problemas sociales. Por último, se realizó una actividad grupal donde los alumnos crearon una intervención comunitaria en un barrio con altos niveles de desorden físico y otras características negativas del vecindario, y posteriormente presentaron su proyecto de forma oral llevando a cabo estrategias de márketing social. Los alumnos mostraron un gran interés en la creación de sus propios proyectos, y las presentaciones grupales de los proyectos fueron de una alta calidad.

\section{Conclusiones}

En este trabajo se ha presentado una práctica docente basada en el paradigma. Research Based Learning, la cual propone el aprendizaje a través de la curiosidad científica. Esta práctica docente va más allá de la enseñanza tradicional y presenta el Aprender haciendo como una alternativa que implica al alumnado en su propio aprendizaje, acercándole a la investigación científica en el marco de su propia disciplina académica.

La valoración que el equipo docente ha llevado a cabo respecto a la actividad ha sido muy positiva. Esta actividad no sólo ha tenido muy buena acogida por parte de los y las estudiantes, sino que les ha resultado una oportunidad para aprender a utilizar nuevas herramientas tecnológicas y potenciar sus capacidades de aprendizaje autónomo. Asimismo, los y las estudiantes valoraron muy positivamente la retroalimentación propuesta por el equipo docente y la posibilidad de discutir y compartir los resultados que habían obtenido en su investigación tanto con sus compañeros como con los propios docentes.

Este ha sido el primer año en el que se ha aplicado esta práctica en la asignatura de Psicología de la Intervención Social y Comunitaria de forma sistemática, y nuestro objetivo es continuar realizándola durante los próximos años con el fin de evaluar científicamente la satisfacción de los alumnos/as, la percepción de los profesores, así como el cambio de actitudes hacia la investigación una vez acabada la asignatura. En futuros estudios se prevé proporcionar resultados basados en el rigor científico sobre la efectividad de la aplicación de esta práctica en la asignatura de referencia.

\section{Agradecimientos}

Este proyecto ha sido realizado en el marco de la convocatoria de innovación del Vicerectorat de Polítiques de Formació i Qualitat Educativa de la Universitat de València (UV-SFPIE_RMD16-417684). 


\section{Referencias}

BADLEY, G. (2002). "A really useful link between teaching and research" en Teaching in Higher Education, vol. 7, issue 4, p. 443-455.

ELTON, L. (2001). "Research and teaching: what are the real relationships?" en Teaching in Higher Education, vol. 6, issue 1, p. 43-56.

GRIFFITS, R. (2004). "Knowledge production and the research-teaching nexus: the case of the built environments disciplines" en Studies in Higher Education, vol. 29, issue 6, p. 709-726.

HEALEY, M., y JENKINS, A. (2009). Developing Undergraduate Research and Inquiry. York: HE Academy.

HUTCHINGS, W. (2007). Enquiry-Based Learning: Definitions and Rational. Manchester: University of Manchester.

JENKINS, A. (2004). A guide to the research evidence on teaching-research relationships. York: Higher Education Academy.

LEE, R. (2004). "Research and teaching: making - or breaking - the links" en Planet, vol. 12 , p. 9-10.

MARCO, M., GRACIA, E., TOMÁS, J.M., y LÓPEZ-QUíLEZ, A. (2015). “Assessing neighborhood disorder: Validation of a three-factor observational scale" en The European Journal of Psychology Applied to Legal Context, vol. 7, issue 2, p. 81-89.

ODGERS, C.L., CASPI, A., BATES, C.J., SAMPSON, R.J. y MOFFIT, T.E. (2012). "Systematic social observation of children's neighborhoods using Google Street View: a reliable and cost-effective method" en Journal of Child Psychology and Psychiatry, vol. 53 , issue 10 , p. 1009-1017. 